\title{
The Effects of Biarum carduchrum Hydroalcoholic Extract on Oxidative Stress and Catalepsy in 6-hydroxydopamine-induced Rat Model of Parkinson's Disease
}

\author{
Mahbubeh Setorki $^{1}$ (D) Zahra Hooshmandi ${ }^{\text {iD }}$, Zahra Zanganehnejad ${ }^{3 *}$ \\ 1. Dept. of Biology, Izeh Branch, Islamic Azad University, Izeh, Iran \\ 2. Dept. of Biology, Sanandaj Branch, Islamic Azad University, Sanandaj, Iran \\ 3. Dept. of Biology, Shahrekord Branch, Islamic Azad University, shahrekord, Iran
}

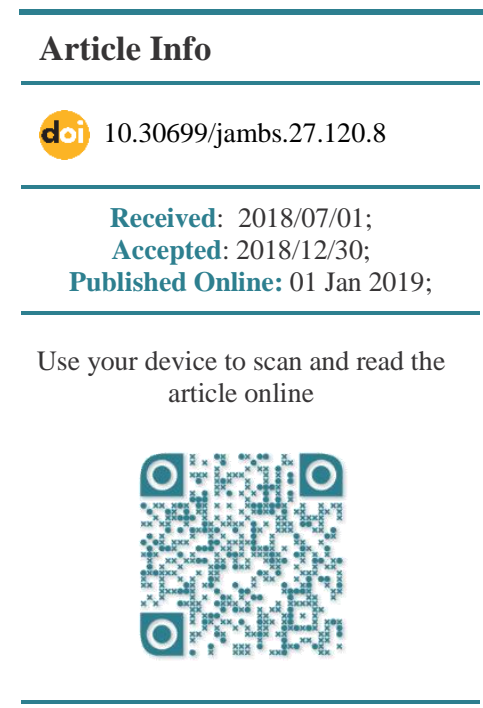

Corresponding Information: Zahra Zanganehnejad, Dept. of Biology, Shahrekord Branch, Islamic Azad University, shahrekord, Iran Email: zzangenehnejad@yahoo.com

\begin{abstract}
Background \& Objective: Parkinson is a neurodegenerative disease that leads to incurable and debilitating conditions. Herbal extracts can afford protection against neurodegenerative diseases due to their bioactive compounds. In the present study, we investigated the effect of hydro-alcoholic extract of Biarum carduchrum on catalepsy and brain oxidative stress in rat's model of Parkinson's disease.
\end{abstract}

Materials \& Methods: Rats were randomly divided into five groups of eight animals. The control group was left intact. Parkinsonian group received an injection of 6hydroxydopamine (6-OHDA) in the right anterior mid-brain. Extract treated groups received hydro-alcoholic extract of $B$. carduchrum at doses of 100,200 and $400 \mathrm{mg} / \mathrm{kg}$ by gavage seven days after 6-OHDA injection. 14 days after treatment, bar test was performed and lipid peroxide levels of different brain regions were determined. Data were analyzed by ANOVA followed by Tukey's test using SPSS22 software and $P<0.05$ was considered statistically significant.

Results: In 6-OHDA-lesioned group bar time was increased significantly $(P<0.05)$ when compared with the control group $(122.50 \pm 90.12$ versus $0.00 \pm 0.00)$. $B$. carduchrum at doses of 200 and $400 \mathrm{mg} / \mathrm{kg}$ significantly reduced 6-OHDA induced catalepsy $(P<0.0 .5)$. 6-OHDA treatment lead to significant increases in lipid peroxide levels of cerebellum, cortex, hippocampus and striatum $(P<0.05)$. Administration of $B$. carduchrumextract at different doses caused significant reduction in the lipid peroxide levels of different brain regions $(P<0.05)$.

Conclusion: B. carduchrum extract ameliorated 6-OHDA -induced catalepsy and lipid peroxide level of brain in rat's model of Parkinson's disease.

Keywords: Biarum carduchrum, Catalepsy, Parkinson's disease, Lipid peroxide

\section{Introduction}

Parkinson's disease is a neurodegenerative disease that occurs mainly due to the progressive destruction of dopaminergic neurons in Substantia nigra, pars compacta $(\mathrm{SNc})$ and other regions of the brainstem (1). Dopamine plays a critical role in the movement and motor functions. Acetylcholine is another neurotransmitter that opposes the action of dopamine. In Parkinson's disease, dopaminergic neurons are dying and therefore the balance between the dopaminergic and the cholinergic activity is disturbed, which results in the development of disease symptoms including tremor, bradykinesia, catalepsy, and dystonia (2). Catalepsy is a physical condition characterized by muscular rigidity, fixity of posture, suspension of sensation and loss of contact with environment (3). Other symptoms of the disease include cognitive impairment and speech disorder (4).

Oxidative Stress has been reported to play an important role in the degeneration of dopaminergic neurons in
Parkinson's disease (5). Oxidative stress in Parkinson disease arises from elevated iron accumulation, impaired mitochondrial function, increased nitric oxide production and weakened antioxidant defense system. Overproduction of reactive oxygen species can lead to oxidative damage of cellular biomolecules and eventually dopaminergic cell death (6). Oxidative stress can also lead to dopaminergic cell damage by disturbing the process of oxidative phosphorylation and energy depletion (7).

There is currently no cure for the treatment of Parkinson's disease. Some drugs can help alleviate the symptoms of the disease but exert minimal effects on neuropathological changes caused by the disease. In addition, some of these drugs show serious side effects and aren't tolerated by the patient. Therefore, there is a clinical need for new therapeutic agents to stop or slow down the neurodegenerative process in the pathology of disease (8). 
Biarum is a genus of about 20 species of flowering plants in the family Araceae that are native to the Middle East, southern Europe, and North Africa (9). Biarum carduchrum is a medicinal plant species from this genus and grows wild in Turkey, Syria, Lebanon, Jordan, Iraq and Iran. In Iran, B. carduchrum is found on the hillsides of the Zagros Mountains in Fars and Kohgiluyeh and Boyer-Ahmad Provinces (10). There is very limited information about the pharmaceutical effect of this plant. In a study, $B$. carduchrum extract showed potent antioxidant activity in the inhibition of free radicals in vitro. It also showed protective effect against hyperlipidemia induced by high-fat diets in rat model (11). In another study, B. carduchrum extract considerably enhanced the antioxidant status of rat brain tissue by improving its thiol level (10). The species of Biarum genus have been reported to contain alkaloids, amines, saponins, cinnamic acid, and flavonoids (9). Due to their potent antioxidant effects, these compounds can protect the body against the harmful effects of free radicals and active oxygen radicals (12). Regarding the antioxidant property of $B$. carduchrum $(\mathbf{1 0}, 11)$, we investigated the effects of hydro-alcoholic extract of this plant on animal catalepsy and lipid peroxidation of different brain regions in rat model of Parkinson's disease.

\section{Materials and Methods}

\section{Preparation of Hydroalcholic Extract of B. carduchrum}

B. carduchrum samples were collected from the Izeh vicinity, Khuzestan Province, South-West Iran during spring 2018. After identification by a botanist, the plant specimens were stored in the herbarium of Islamic Azad University of Izhe (Number: 45679). Then, the leaves were separated and dried in the open air under shadow. The dried leaves were finely pulverized into particles less than $0.4 \mathrm{~mm}$ in diameter. The resulting powder was macerated with $70 \% \mathrm{v} / \mathrm{v}$ ethanol for $72 \mathrm{~h}$ at room temperature. Then, the resulting extract was filtered and the filtrate underwent vacuum evaporation to remove alcohol (the extract yield was calculated 28\%) (13).

\section{Grouping of Animals:}

Male Wistar rats weighing 200-250g were housed under standard conditions $\left[(21 \pm 2)^{\circ} \mathrm{C}\right.$ temperature and 12-h light-dark cycle] with free access to the same water and food. The rats were assigned into five groups with eight rats in each. The Control group was left intact. Parkinsonian group received injection of 6OHDA in the right anterior mid-brain (14). Extract treated groups received B. carduchrum extract at doses of 100,200 and $400 \mathrm{mg} / \mathrm{kg}$ by gavage for 14 days, seven days after Parkinson induction $(15,16)$. The rats were given 7 days to heal their wound, and then the treatment began. Doses of the extract was selected based on previous studies $(10,11)$. After treatment period, behavioral test was performed. The study was approved by the Ethics Committee of Islamic Azad University (Cod: IR. IAU. Ahwaz. REC. 1395.53) and all animal procedure was based on Guidelines for the Care and Use of Laboratory Animals (17).

\section{Inducing of Parkinson's Disease}

The rats were anesthetized using an IP injection of ketamine hydrochloride at $90 \mathrm{mg} / \mathrm{kg}$ plus xylazine at 10 $\mathrm{mg} / \mathrm{kg}$. Then, the rats were placed in a stereotaxic apparatus and stabilized by a mouthpiece and the rods inside the headphone and the hairs in the back of its cranium was shaved. The rat head skin was fumigated with alcohol cotton and a linear section was made from the back of the head between the two eyes to the midpoint of the back of the middle ears. The connective tissues on the cranium surface were removed and the bregma was displayed. The bregma and lambda were placed on an equal surface and the apparatus marker was set on it. Correspondent to Atlas of Neurosurgical Techniques, the MFB was defined at peculiarities AP:3.8, ML \pm 1.8 , and DV:-3.8mm. Parkinson's disease was enforced by unilaterally injecting neurotoxin-6-OHDA ( $8 \mu \mathrm{g}$ ) (dissolved in $2 \mu \mathrm{g}$ of normal saline containing $1 \%$ ascorbic acid) in the MFB (Figure 1) (14).

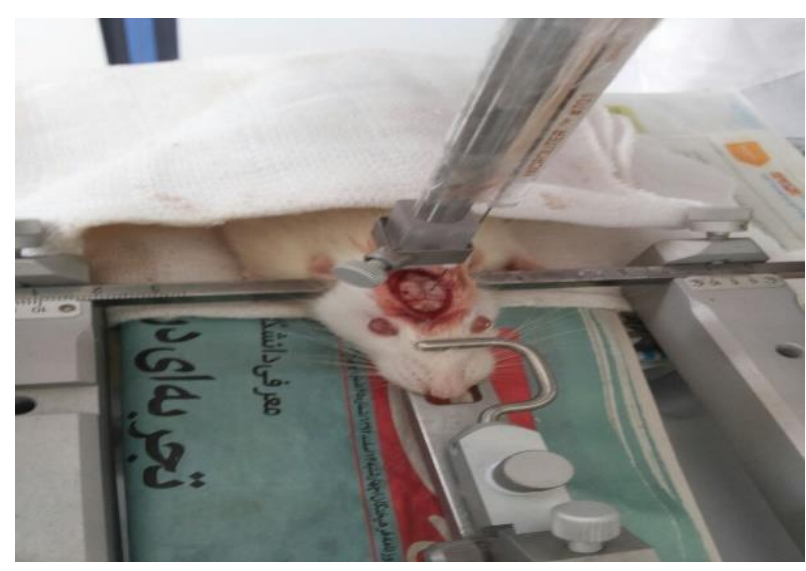

Figure 1. Finding the positions of Substantia Nigra

\section{Bar Test (Catalepsy)}

In the present study, bar test was used to evaluate the 6OHDA induced catalepsy. The device used in this test was a wooden bar with a platform. The height of the floor from the platform was $9 \mathrm{~cm}$ and the diameter of the horizontal bar was $0.9 \mathrm{~cm}$. To perform the experiment, the animal was placed on the platform and its two front paws were placed lightly on the horizontal bar. The duration of time animal remained in this inflicted condition was discussed as the bar time. The end point of catalepsy was defined to occur when both front paws were lifted from the bar or if the animal moved its head in a probative method. The cut off time of the test was $720 \mathrm{sec}$ and the test was done in four continuous times with $1 \mathrm{~h}$ interval (18).

\section{Measuring Lipid Peroxide Levels in the Brain Tissues}

The rats' brains were placed in a plate containing normal saline which was cooled on ice and then the brain regions including cerebellum, hippocampus, striatum and cortex were separated by scalpel and immediately weighed. One gram of each section was homogenized in $10 \mathrm{~mL}$ of cold $1.5 \% \mathrm{KCl}$ solution. Then, $0.5 \mathrm{~mL}$ of homogenized sample, $3 \mathrm{~mL}$ of $1 \%$ phosphoric acid and $1 \mathrm{~mL}$ and $1 \mathrm{~mL}$ 
of $6 \%$ thiobarbituric acid were added to a test tube and heated in a boiling water bath for $45 \mathrm{~min}$. After cooling, 4 $\mathrm{mL}$ of $\mathrm{n}$-butanol was added and mixed vigorously. After centrifugation at $2000 \mathrm{rpm}$ for 15 minutes, the absorbance read at $535 \mathrm{~nm}$ wavelength (19).

\section{Statistical analysis}

Data analysis was conducted by SPSS version 22 . First, normal distribution of the data was investigated by Kolmogorov-Smirnov test and variance homogeneity was studied by Levene's test. Then, to investigate the significance of difference between the treatments, oneway ANOVA was used, and to compare the mean values, Tukey's test. The data were expressed as mean (standard. deviation) and $\mathrm{p}<0.05$ was considered statistically significant.

\section{Results}

The effect of hydro-alcoholic extract of $B$. carduchrum on 6-OHDA induced catalepsy in the bar test was shown in Table 1. Injection of 6-OHDA into the rat brain caused a significant increase in the bar time compared to the control group $(P<0.05)$. Treatment of 6OHDA-lesioned rats with $B$. carduchrum extract at doses of 200 and $400 \mathrm{mg} / \mathrm{kg}$ significantly reduced the bar time compared to the 6-OHDA group $(P<0.05)$. Mean bar time in 6-OHDA-lesioned group receiving extract at a doses of $100 \mathrm{mg} / \mathrm{kg}$ was significantly higher than group receiving extract at doses of 200 and 400 $\mathrm{mg} / \mathrm{kg}(P<0.05)$. Mean bar time in 6-OHDA-lesioned groups receiving extract at doses of 200 and $400 \mathrm{mg} / \mathrm{kg}$ had no significant difference (Table 1 ).

The effect of B. carduchrum extract at doses of 100 , 200 , and $400 \mathrm{mg} / \mathrm{kg}$ on lipid peroxide levels in different brain regions of rats was shown in Figure 2. There was a statistically significant difference in the lipid peroxide levels of cerebellum, cortex, hippocampus and striatum tissues between the control and 6-OHDA-lesioned groups $(P<0.05)$. Treatment of 6-OHDA-lesioned group with $B$. carduchrum extract (at all doses) caused significant decreases in lipid peroxide levels of the all brain regions $(P<0.05$, Figure 1). B. carduchrum extract at $100 \mathrm{mg} / \mathrm{kg}$ showed better activity in lowering brain lipid peroxide compared to doses of 200 and $400 \mathrm{mg} / \mathrm{kg}$.

Table 1. The effect of B. carduchrum hydroalcoholic extract on 6-OHDA induced catalepsy in the bar test

\begin{tabular}{ccc}
\hline Experimental group & $\begin{array}{c}\text { Bar time (seconds) } \\
\text { Mean+SD }\end{array}$ & P values \\
\hline 6-OHDA-lesioned group & $122.50 \pm 9.02$ & \\
Control & $00.00 \pm 00.00^{*}$ & 0.05 \\
Extract $(100 \mathrm{mg} / \mathrm{kg})$ & $93.66 \pm 5.45$ & \\
Extract $(200 \mathrm{mg} / \mathrm{kg})$ & $22.00 \pm 1.35^{\# \&}$ & \\
Extract $(400 \mathrm{mg} / \mathrm{kg})$ & $29.33 \pm 2.8^{\# \&}$ & \\
\hline
\end{tabular}

Data were analyzed by one-way ANOVA followed by Tukey's test. *Shows significant difference between 6-OHDA-lesioned and control groups at $P<0.05$. " Shows significant difference between 6-OHDA-lesioned and extract treated groups at $P<0.05$. \& show significant different between 6OHDA-lesioned group receiving extract at a dose of $100 \mathrm{mg} / \mathrm{kg}$ with 6-OHDA-lesioned groups receiving extract at doses of $200 \mathrm{and} 400 \mathrm{mg} / \mathrm{kg}$.

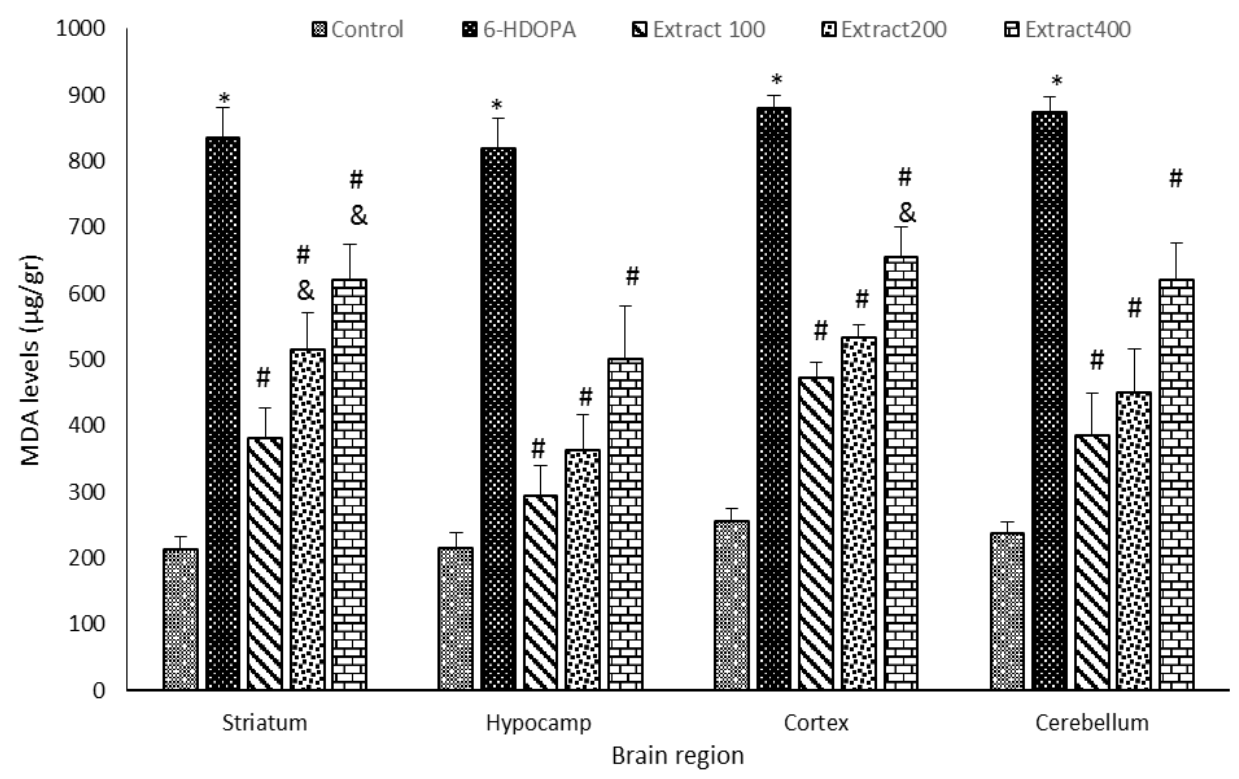

Figure 2. The effect of Biarum carduchrum extract at doses of 100, 200, and 400mg/kg on lipid peroxide levels of different brain region; *Shows significant difference between 6-OHDA-lesioned and control groups at $P<0.05$. ${ }^{*}$ shows significant difference between 6-OHDA-lesioned and extract treated groups, \& show significant different between 6-OHDA-lesioned group receiving extract at a dose of $100 \mathrm{mg} / \mathrm{kg}$ with 6-OHDA-lesioned groups receiving extract at doses of $200 \mathrm{and} 400 \mathrm{mg} / \mathrm{kg}$. 


\section{Discussion}

Our results showed that 6-OHDA injection was able to cause catalepsy, this result is consistent with previous studies reporting catalepsy inducing activity for 6-OHDA $(15,20)$. Generally, 6-OHDA is a neurotoxic agent used to induce nigrostriatal damage and establish an animal model of Parkinson's disease. It cannot pass the blood brain barrier, so it must be directly injected in the SNc (20). It was shown that progressive degeneration of dopaminergic neurons of SNc and other brain regions due to 6-OHDA toxicity results in motor disabilities such as muscle rigidity (catalepsy), akinesia, tremor and postural abnormalities (15).

In our study, intra-striatal injection of 6-OHDA caused significant increases in lipid peroxides levels of different brain regions. Previous studies have also reported different degrees of oxidative stress following intrastriatal administration of 6-OHDA. Haddadi et al. and Hosseini et al. reported rise of brain lipid peroxidation in 6-OHDA-lesioned rats (21). Khan et al reported elevated levels of thiobarbituric acid reactive substances (TBARS) and protein carbonyl and reduced activities of antioxidant enzyme in different brain regions of parkinsonian rats (22).

It has been well established that neurotoxin-6-OHDA causes degeneration of the brain areas involved in motor function by producing free radicals and reactive oxygen species. Under physiological conditions, this toxin is readily oxidized and converted to hydrogen peroxide and, through a specific reaction, hydroxyl radicals, which are one of the most harmful free radicals for living cells (23, 24). Hydroxyl radical and other reactive oxygen species can cause oxidative damage to biologically vital molecules such as lipids, proteins and DNA which ultimately leads to neuronal cell injury and death (25). Brain tissues contain a high amount of unsaturated fatty acids that are particularly vulnerable to free radicals' attack. It is argued that lipid peroxidation is a predominant and specific type of neuronal oxidative stress that damages the cell membrane and produces secondary metabolites which show neurotoxic effects (26).

Our results showed that the treatment of 6-OHDAlesioned rats with $B$. carduchrum extract at different doses significantly suppressed the rise in lipid peroxidation of brain areas. There is limited information about $B$. carduchrum and very few studies have been conducted to determine the phytochemical composition and biological activities of this plant. The study of Hosseini et al. indicated that $B$. carduchrum extract acts similarly with or even more potently than certain nature-derived or synthetic antioxidants in vitro. They also investigated the activity of hydromethanolic extract of B. carduchrum on the serum lipids in rats and reported that this extract could improve fat-rich diet-induced increase in serum lipids due to potent antioxidant property (11). In another study conducted by Zanganehnejad et al., it was found that treatment with $B$. carduchrum extract significantly increases antioxidant potential in the different brain regions of parkinsonian rats (10).

Potent products derived from plants, fruits, and vegetables exert considerable antioxidant effects against neuronal oxidative stress due to certain compounds such as flavonoids, steroidal lactones, alkaloids, caffeine, saponins, anthocyanins, and curcuminoid (27). The plants of Biarum genus can synthesize many compounds such as flavonoids, phenols, alkaloids, amines and saponins (9). Regarding the protective effects of these compounds on oxidative stress-induced neuronal injuries (28-31), it can be argued that the antioxidant compounds of $B$. carduchrum, including phenolic and flavonoids, may prevent the progression of nervous system neurodegeneration through reducing oxidative stress parameters.

In this study, B. carduchrum extract at $100 \mathrm{mg} / \mathrm{kg}$ showed better activity in lowering brain lipid peroxidation than 200 and $400 \mathrm{mg} / \mathrm{kg}$ doses. It has also been observed that some herbal extracts that contain high levels of polyphenols may exacerbate oxidative stress by interfering with oxidative-antioxidant balance (22). So, it seems that lower effects of 200 and $400 \mathrm{mg} / \mathrm{kg}$ doses of B. carduchrum are related to the imbalance of oxidativeantioxidant status.

\section{Conclusion}

In the present study, for the first time, the supporter effects of B. carduchrum on catalepsy and lipid peroxidation in 6OHDA-lesioned rats were displayed, which may be due to the antioxidant effects of the plant. The supporter effects of this plant may also be ingeminated to the other mechanisms involved in Parkinson pathophysiology which need further study.

\section{Acknowledgements}

This article is the result of student research project number 12947615 Islamic Azad University of Izeh. Code of ethics is IR.IAU.Ahwaz.REC.1396.98.

\section{Conflict of Interest}

Authors declared no conflict of interests. 


\section{References}

1. Jackson-Lewis V, Blesa J, Przedborski S. Animal models of Parkinson's disease. Parkinsonism \& related disorders. 2012; 18 : S183-S5. [DOI:10.1016/S1353-8020(11)70057-8]

2. Bohnen NI, Albin RL. The cholinergic system and Parkinson disease. Behav Brain Res. 2011; 221(2): 564-73. [DOI:10.1016/j.bbr.2009.12.048] [PMID] [PMCID]

3. Ahlskog JE. editor Aerobic exercise: evidence for a direct brain effect to slow parkinson disease progression. Mayo Clinic Proceedings;2018:Elsevier. [DOI:10.1016/j.mayocp.2017. 12.015] [PMID]

4. Hirsch EC, Jenner P, Przedborski S. Pathogenesis of Parkinson's disease. Movement Disorder. 2013; 28(1): 24-30. [DOI:10.1002 /mds.25032] [PMID]

5. Zhou C, Huang Y, Przedborski S. Oxidative stress in Parkinson's disease. Ann New York Acad Sci. 2008; 1147(1): 93-104. [DOI:10.1196/annals.1427.023] [PMID] [PMCID]

6. Hwang O. Role of oxidative stress in Parkinson's disease. Experiment Neurobiol. 2013; 22(1): 11-7. [DOI:10.5607/ en.2013.22.1.11] [PMID] [PMCID]

7. Henchcliffe C, Beal MF. Mitochondrial biology and oxidative stress in Parkinson disease pathogenesis. Nature Rev Neurol. 2008; 4(11): 600-9. [DOI:10.1038/ncpneuro0924] [PMID]

8. Jadiya P, Khan A, Sammi SR, Kaur S, Mir SS, Nazir A. AntiParkinsonian effects of Bacopa monnieri: insights from transgenic and pharmacological Caenorhabditis elegans models of Parkinson's disease. Biochem Biophysic Res Commun. 2011; 413(4): 605-10. [DOI:10.1016/j.bbrc.2011.09.010] [PMID]

9. Farahmandfar R, Ramezanizadeh MH. Oxidative stability of canola oil by Biarum bovei bioactive components during storage at ambient temperature. Food Sci Nutr. 2018; 6: 342-47. [DOI:10.1002/fsn3.560] [PMID] [PMCID]

10. Zanganehnejad Z, Setorki M. Effect of Biarum carduchrum extract on brain tissue thiol level in rat model of 6hydroxydopamine-induced Parkinson's disease. J Herb Med Pharmacol. 2018; 7(3): 136-40. [DOI:10.15171/jhp.2018.23]

11. Hosseini E, Rousta E, Tabib Loghmany F, Mahmoudpour M. In vitro antioxidant activity of hydromethanolic extract of Karde (Biarum carduchrum) and tts effects on the serum lipids of rats. Iran J Nut Sci Food Technol. 2014; 9(3): 1-8.

12. Pisoschi AM, Pop A. The role of antioxidants in the chemistry of oxidative stress: A review. Europ J Med Chem. 2015; 97: 55-74. [DOI:10.1016/j.ejmech.2015.04.040] [PMID]

13. SeifiZangeneh M, Rafieirad M, Sazgar H. The effect of Kardeh (BiarumBovei) hydro-alcoholic extract on pain threshold in STZ induced diabetic rats. J Herb Drug. 2015; 6(3): 137-42.

14. Simola N, Morelli M, Carta A. The 6-hydroxydopamine model of Parkinson'sdisease. Neurotoxic Res. 2008; 11(1): 151-67. [DOI:10.1007/BF03033565] [PMID]

15. Rafieiradorcid M, Doulahorcid A, Rostami SA. Effects of strawberry (Fragaria ananassa) extract on 6-hydroxy dopamine induced parkinson's disease model in male rats. Report Health Care J. 2016; 2(3): 1-8.

16. Goudarzi S, Rafieirad M. Evaluating the effect of $\alpha$-pinene on motor activity, avoidance memory and lipid peroxidation in animal model of Parkinson disease in adult male rats. Res $\mathrm{J}$ Pharmacog. 2017; 4(2): 53-63.
17. Olfert ED, Cross BM, McWilliam AA. Guide to the care and use of experimental animals: Canadian Council on Animal Care Ottawa; 1993.

18. Salar F, Ziai S, Nasri S, Roghani M, Kamalinejad M. Neuroprotective effect of aqueous extract of Berberis vulgaris $\mathrm{L}$. in a model of parkinson's disease in rat. J Med Phys. 2010; 4(36): 24-33.

19. Mansouri SMT, Naghizadeh B, Hosseinzadeh H. The effect of Pistacia vera L. gum extract on oxidative damage during experimental cerebral ischemia-reperfusion in rats. Iran Biomed J. 2005; 9(4): 181-5.

20. Kheradmand A, Nayebi AM, Jorjani M, Haddadi R. Effect of WR-1065 on 6-hydroxydopamine-induced catalepsy and IL-6 level in rats. Iran J Basic Med Sci. 2016; 19(5): 490-6.

21. Haddadi R, Nayebi AM, Farajniya S, Brooshghalan SE, Sharifi H. Silymarin improved 6-OHDA-induced motor impairment in hemi-parkisonian rats: behavioral and molecular study. DARU. 2014; 22(1): 38. [DOI:10.1186/2008-2231-22-38] [PMID] [PMCID]

22. Khan MM, Ahmad A, Ishrat T, et al. Resveratrol attenuates 6hydroxydopamine-induced oxidative damage and dopamine depletion in rat model of Parkinson's disease. Brain Res. 2010; 1328: 139-51. [DOI:10.1016/j.brainres.2010.02.031] [PMID]

23. Kim GH, Kim JE, Rhie SJ, Yoon S. The role of oxidative stress in neurodegenerative diseases. Exp Neurobiol. 2015; 24(6): 80412. [DOI:10.5607/en.2015.24.4.325] [PMID] [PMCID]

24. Sarrafchi A, Bahmani M, Shirzad H. Oxidative stress and Parkinson's disease: New hopes in treatment with herbal antioxidants. Curr Pharmaceut Design. 2016; 2(2): 161S-70S. [DOI:10.2174/1381612822666151112151653] [PMID]

25. Bhat A, Dar K, Anees S, Zargar M. Oxidative stress, mitochondrial dysfunction and neurodegenerative diseases; a mechanistic insight. Biomed Pharmacother. 2015; 7(33): 10110. [DOI:10.1016/j.biopha.2015.07.025] [PMID]

26. Montine TJ, Neely MD, Quinn JF, et al. Lipid peroxidation in aging brain and Alzheimer's disease1, 2. Free Radical Biology and Medicine. 2002; 33(5): 620-6. [DOI:10.1016/S08915849(02)00807-9]

27. Lee SE, Hwang HJ, Ha J-S, Jeong H-S, Kim JH. Screening of medicinal plant extracts for antioxidant activity. Life Sci. 2003; 73(2): 167-79. [DOI:10.1016/S0024-3205(03)00259-5]

28. Ishige K, Schubert D, Sagara Y. Flavonoids protect neuronal cells from oxidative stress by three distinct mechanisms. Free Radical Biol Med. 2001; 30(4): 433-46. [DOI:10.1016/S08915849(00)00498-6]

29. Soobrattee MA, Neergheen VS, Luximon-Ramma A, Aruoma OI, Bahorun T. Phenolics as potential antioxidant therapeutic agents: mechanism and actions. Mutat Res. 2005; 579(1): 200-13. [DOI:10.1016/j.mrfmmm.2005.03.023] [PMID]

30. Sultana R, Ravagna A, Mohmmad-Abdul H, Calabrese V, Butterfield DA. Ferulic acid ethyl ester protects neurons against amyloid $\beta$-peptide (1-42)-induced oxidative stress and neurotoxicity: relationship to antioxidant activity. J Neurochemist. 2005; 92(4): 749-58. [DOI:10.1111/j.14714159.2004.02899.x] [PMID]

31. Mansouri MT, Farbood Y, Sameri MJ, Sarkaki A, Naghizadeh B, Rafeirad M. Neuroprotective effects of oral gallic acid against oxidative stress induced by 6-hydroxydopamine in rats. Food Chem. 2013; 138(2-3): 1028-33. [DOI:10.1016/j.foodchem 2012.11.022] [PMID] 


\section{How to Cite This Article:}

Setorki M, Hooshmandi Z, Zanganehnejad Z. The effects of Biarum carduchrum hydroalcoholic extract on oxidative stress and catalepsy in the 6-hydroxydopamine-induced rat model of Parkinson's disease. J Adv Med Biomed Res. 2019; 27 (120) :8-13

Download citation:

BibTeX | RIS | EndNote | Medlars | ProCite | Reference Manager | RefWorks

Send citation to:

Mendeley 2 Zotero :i)efWorks $\underline{\text { RefWorks }}$ 